\title{
ERRATUM
}

\section{D-printed microelectronics for integrated circuitry and passive wireless sensors}

Sung-Yueh Wu, Chen Yang, Wensyang Hsu and Liwei Lin

Microsystems \& Nanoengineering (2015) 1, 15036; doi:10.1038/micronano.2015.36; Published online: 9 November 2015

Erratum to: Microsystems \& Nanoengineering (2015) 1, 15013; doi: 10.1038/micronano.2015.13; Published online: 20 July 2015

Since the publication of this article, the authors would like to make two corrections in the third(last) paragraph of "Design and Fabrication" section as follows:

1 Replace "fused deposition modeling technology" with "multi-jet modeling (MJM)";

2 The company information of "ProJet HD 3000 printer, Hewlett-Packard Company, Palo Alto, CA, USA" should be replaced with "ProJet HD 3000 printer, 3D Systems Inc., Rock Hill, SC, USA".

We apologize for any inconvenience may have caused. 